\title{
Instability control in microwave-frequency microplasma
}

\author{
N. Miura and J. Hopwood ${ }^{\mathrm{a}}$ \\ Electrical and Computer Engineering, Tufts University, Medford, 02155 Massachusetts, USA
}

Received 17 December 2011 / Received in final form 28 March 2012

Published online 23 May 2012

(C) The Author(s) 2012. This article is published with open access at Springerlink.com

\begin{abstract}
Atmospheric argon microplasmas driven by $1.0 \mathrm{GHz}$ power were studied by microwave circuit analyses and spatially-resolved optical diagnostics. These studies illuminate the mechanisms responsible for microplasma stability. A split-ring resonator (SRR) microplasma source is demonstrated to reflect excess microwave power, preventing the ionization overheating instability while limiting electron density to approximately $1 \times 10^{14} \mathrm{~cm}^{-3}$ and $\mathrm{OH}$ rotational temperature to $760 \mathrm{~K}$ at $0.76 \mathrm{~W}$. Providing the SRR microplasma with an electrical path to ground, however, allows the microplasma to transition from the SRR mode to the so-called transmission line mode (T-line). This transition is due to matching of the microplasma and transmission line impedances. The higher power T-line mode supports a more intense microplasma with electron density of $1 \times 10^{15} \mathrm{~cm}^{-3}$ and $\mathrm{OH}$ rotational temperature of $1480 \mathrm{~K}$ with $15 \mathrm{~W}$ absorbed power. While the SRR mode is optimized for ignition and sustaining a stable nonequilibrium plasma, and T-line mode is better suited for driving a hot, high density microplasma. The estimated microwave discharge voltages were $15 \mathrm{~V}$ and $35 \mathrm{~V}$ in SRR mode and T-line mode, respectively, and the voltages are rather independent of input power. Microplasma stability is due to a combination of impedance mismatching and direct control of power, both inherent to microwave circuitry.
\end{abstract}

\section{Introduction}

Atmospheric microplasmas are normally classified as nonequilibrium plasmas, that is, the electron temperature $(\sim 10000 \mathrm{~K})$ is much higher than the gas temperature which is typically less than $1000 \mathrm{~K}$. The non-thermal, nondestructive nature of microplasmas suggests that these discharges may be useful in biomedical applications, surface modification of plastics, miniaturized sensors and labon-a-chip.

Many microplasma sources are driven by direct current (DC) $[1-6]$ or pulsed DC power supplies $[7,8]$. Microwave microplasma sources [9-20], while more complex to design, can provide some tangible benefits. For example, microwave-driven electrodes can sustain a discharge with lower potentials, which in turn reduces sputtering of the electrodes and significantly prolongs the lifetime of the device. The smaller sheath voltage also reduces ion Joule heating of the gas allowing more power to be partitioned into electron heating. Although large-scale microwave plasmas are often electrodeless, these two properties allow microplasmas to be efficiently driven directly from microfabricated electrodes.

Plasma instabilities are often a concern, and because microplasmas are operated near atmospheric pressure the ionization overheating instability (IOI) must be controlled. Staack provides a thorough analysis of this instability in the context of DC microdischarges [21]. In brief,

\footnotetext{
a e-mail: hopwood@ece.tufts.edu
}

the instability is caused by electron collisions which heat the neutral gas. The reduction in gas density $(N)$ due to heating increases the reduced electric field $(E / N)$. If the electric field is not decreased in some manner, the electron mean energy increases as specified by the Boltzmann equation. Higher electron energy increases the ionization rate, leading to more electrons and a further increase in gas heating. This unstable situation is one cause of the glow-to-arc transition. In DC microplasmas, one places a ballast resistor in series with the discharge such that an increase of discharge current reduces the discharge voltage. A sufficiently large ballast resistance may prevent the IOI and the formation of a destructive arc. Several investigators have reported, however, that parasitic capacitance within the system may store sufficient energy to temporarily drive the DC microplasma into a high-density state [21-23]. These unstable self-oscillations can be deleterious to the plasma process or the plasma generator.

Another common method of controlling microdischarges is to employ short pulses of DC power $[7,8]$. This can be readily accomplished using a nanosecond pulsed power supply. Alternatively, stability is achieved by coating at least one electrode with an insulating layer and applying an AC potential. Once the discharge is initiated, surface charging of the insulator limits the electric field inside the plasma, extinguishing the ionization rate within a microsecond or less [24-28] The duration of the discharge is less than the IOI time constant. The dielectric barrier discharge (DBD) is quite widely used due to this 
simple and effective stabilization of the IOI. The DBD, however, is transient in nature and the low duty-cycle $(\sim 1 \%)$ limits the time-averaged electron density to the order of $1 \times 10^{11} \mathrm{~cm}^{-3}[28,29]$.

Previously, we have studied microplasma sources based on a microstrip split-ring resonator (SRR) [10-14,30-32]. Measurements of this plasma have thus far suggested that no instabilities exist. This manuscript describes simulations and diagnostics that determine the mechanism responsible for controlling the IOI in microwave discharges. We then modify the SRR to include an electrical path between the microplasma and ground. In this new configuration a hot, dense mode of operation is discovered and characterized.

The SRR source and other microwave resonators are well-suited for plasma ignition because, even with only a watt of power, the unloaded resonator generates an electrode voltage on the order of $200 \mathrm{~V}$. This is sufficient to initiate breakdown in a small discharge gap. The resonator-style source is designed to match the $50 \Omega$ power supply impedance with either no plasma or a relatively high plasma impedance $(>1000 \Omega)$. However, as discharge power is increased and the plasma impedance becomes smaller, the resonator starts to resonate poorly due to resistive loading by the plasma, and the power reflection from the resonator circuit becomes large. While this limits the net power deposition into the plasma to less than a few watts, we show that this also makes the SRR source inherently stable. By placing a ground electrode close to the SRR source, however, the SRR discharge attaches to ground and we can deliver $10 \mathrm{~s}$ of Watts into a plasma. This is shown to be made possible by matching the characteristic impedance of transmission line $(35 \Omega)$ to the highdensity plasma impedance. Throughout the study we refer to this as the transmission line mode (T-line mode). After describing these two different operating modes in detail, this work compares the SRR and T-line modes by microwave circuit analyses and spatially-resolved optical plasma diagnostics.

\section{Experiment and simulation}

\subsection{Plasma source}

An oval-shaped $1.0 \mathrm{GHz}$ microstrip split-ring resonator (SRR) is shown in Figure 1. The copper conductor of the resonator is sandwiched between two sheets of machinable ceramic (Macor) and the back surface of the lower sheet is covered by a copper ground plane. Only the discharge gap formed by the split in the ring is visible in Figure 1b, and so the outline of the ring is marked by a dashed line. The circumference of the 70-ohm transmission line formed by the ring and ground plane is designed to be one-half wavelength at the excitation frequency of $1.0 \mathrm{GHz}$. Microwave power is applied near the top of the ring. The off-center input port is at the location where the SRR has an impedance of $50 \Omega$. In this manner, the voltage amplitude is large across the split at the bottom of the ring due to efficient power coupling and resonance. The high

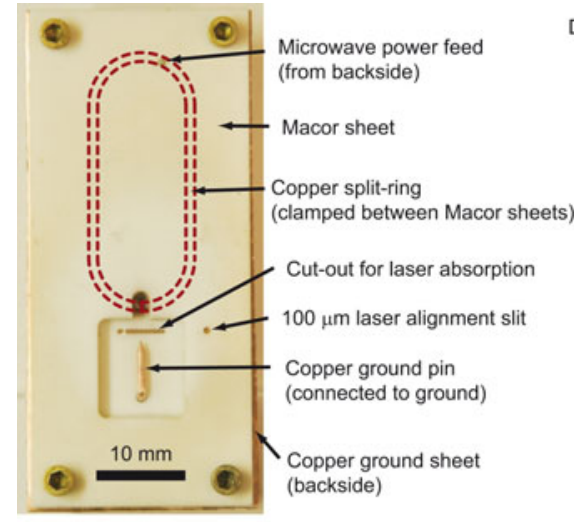

(a)

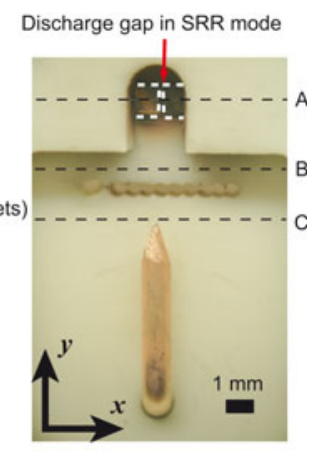

(b)
Fig. 1. (Color online) (a) The modified SRR microplasma source with a $0.1 \mathrm{~mm}$ discharge gap located at the bottom of the oval-shaped ring resonator. (b) A close-up view of the $0.1 \mathrm{~mm}$ discharge gap and copper ground pin. Diagnostics are reported at positions A, B, and C. Line (A) shows the position of the $0.1 \mathrm{~mm}$ discharge gap. Line (C) indicates the grounded electrode position which is $5 \mathrm{~mm}$ below the SRR discharge gap. Spatially resolved measurements of the T-line discharge are made across line (B).

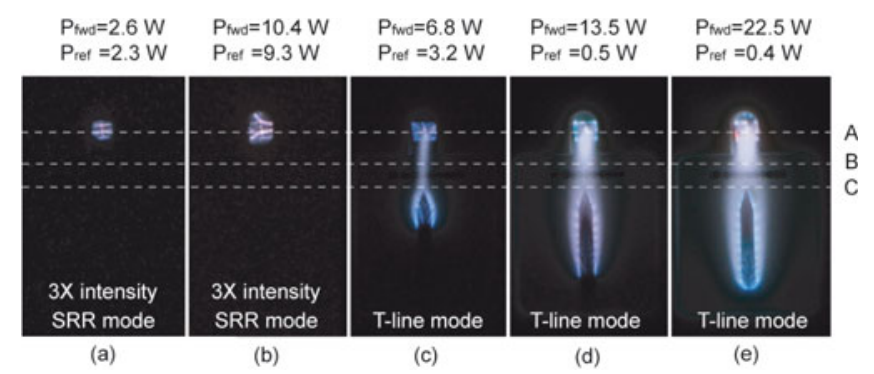

Fig. 2. (Color online) Photographs (a), (b) show the SRR mode discharge within the discharge gap and (c)-(e) show the T-line mode discharge extending to the ground pin (see Fig. 1). The T-line mode initiates in (d) but may be sustained at lower power (c). All microplasmas are operating in argon at one atmosphere with forward and reflected power as noted $(1 \mathrm{GHz})$.

electric field in the discharge gap ignites a microplasma. With relatively small power input, the microplasma is sustained across the split shown in Figures $2 \mathrm{a}-2 \mathrm{~b}$, this is called the split-ring resonator mode (SRR mode). With higher power input, the split in the ring resonator is virtually shorted by the more intense discharge. At these higher powers $(>13 \mathrm{~W})$, the discharge suddenly reforms between the electrodes of the SRR and a ground pin that is intentionally introduced near the SRR discharge (see Figs. 2c-2e). This is called the transmission line mode (T-line mode) and it is sustainable for powers above approximately $4 \mathrm{~W}$. The hysteretic behavior of this mode transition is discussed in Section 3. Note that dashed lines labeled A, B, and $\mathrm{C}$ denote the positions of the SRR discharge gap, the midpoint of the T-line discharge, and the tip of the ground pin, respectively.

The microwave power is fed to the SRR source through a $50 \Omega$ co-axial cable. The forward and reflected microwave powers are measured using a dual-directional coupler and 


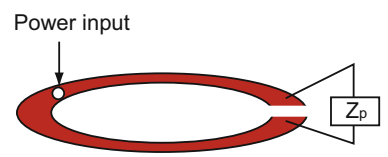

(a) SRR mode

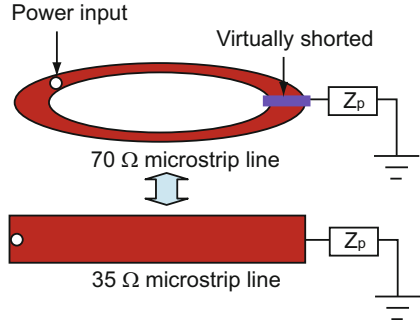

(b) T line mode

Fig. 3. (Color online) Simplified microwave circuit configurations: (a) the SRR mode and (b) the T-line mode. The plasma impedance $\left(Z_{p}\right)$ lies in the discharge gap of the SRR-mode and extends to ground in the T-line mode.

an Agilent power meter. With a microwave circuit, actual power dissipated in the plasma is often difficult to determine accurately. In most cases, the reflected power is simply subtracted from the forward power. However the cable, dielectric, metal and radiative power losses may be too large to neglect. In this work, actual power dissipated is corrected for these losses. Also by comparing both simulated and experimental power reflection coefficients, the plasma resistance, the discharge voltage and current are estimated.

A simplified microwave circuit for each mode is shown in Figure 3. The plasma impedance $\left(Z_{p}\right)$ lies within the discharge gap in Figure 3a. As power is increased, $Z_{p}$ is reduced and the resonator is virtually shorted. The device now acts similar to two 70-ohm transmission lines in parallel (Fig. 3b). Using seed electrons generated from the SRR plasma, a new discharge forms between the nearlyshorted electrodes of the SRR and the ground pin. For this T-line discharge, power is now optimally matched from the 35-ohm equivalent transmission line impedance to a 35-ohm microplasma impedance. Further details of these two modes are described below.

Machinable ceramic $\left(\right.$ Macor $^{\circledR}$, relative permittivity $=$ 5.67, Corning, Inc.) is used as a dielectic substrate of the plasma source. The dielectric material used in our previous work (Duroid ${ }^{\circledR}$, Rogers Corp.) was not able to withstand the higher gas temperatures generated by the T-line mode. Aluminum oxide is the preferred dielectric material, but it is more difficult to machine than Macor. A $100 \mu \mathrm{m}$ thick, $1 \mathrm{~mm}$ wide copper split-ring is sandwiched between $1.77 \mathrm{~mm}$ thick Macor sheets, and a $500 \mu \mathrm{m}$ thick copper sheet was used as an rf ground plane. A $1 \mathrm{~mm}$ diameter copper wire with a sharpened tip penetrates the Macor sheet and is connected to the ground sheet. This pin serves as the ground pin for the T-line mode. The tip of the wire is located $5 \mathrm{~mm}$ from the SRR discharge gap. A $0.5 \mathrm{~mm} \times 5 \mathrm{~mm}$ slot is cut out between the split and the ground pin to create a laser path for diode laser absorption spectrometry. A $100 \mu \mathrm{m}$ copper laser alignment slit is fabricated $5 \mathrm{~mm}$ to the right of this cut-out for diode laser focusing. The entire plasma source is placed inside a vacuum chamber with glass viewports to allow access for the optical diagnostics. The chamber is evacuated by a mechanical pump and a mixture of high purity argon and $0.05 \%$ hydrogen is back filled to near atmospheric pressure. The

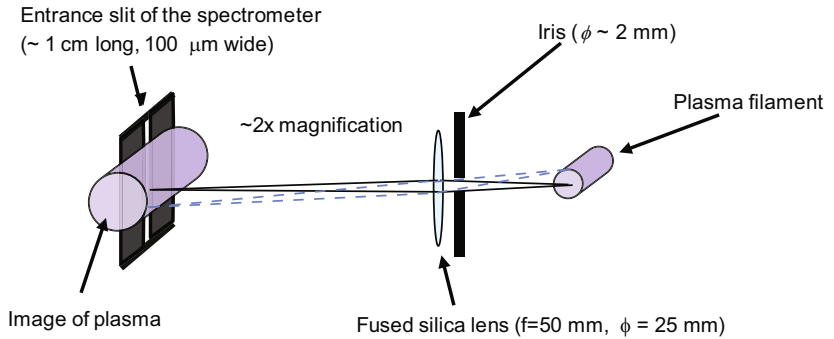

Fig. 4. (Color online) Setup for optical emission spectroscopy. A radial section of the microplasma filament is magnified and focused on the entrance slit of the imaging spectrometer.

actual experimental pressure fluctuates from 750 torr to 800 torr due to gas heating by the T-mode discharge. The substrate, when heated by the plasma, outgasses enough water vapor for $\mathrm{OH}$ emission diagnostics.

\subsection{Optical diagnostic setup}

For measurements of $\mathrm{OH}$ rotational temperature, electron density $\left(\mathrm{H}_{\beta}\right.$ Stark broadening), and excitation temperature, a $0.5 \mathrm{~m}$ imaging spectrometer (Princeton Instruments, 1200 grooves $/ \mathrm{mm}$, blazed at $500 \mathrm{~nm}, 0.1 \mathrm{~nm}$ resolution) and intensified charge-coupled device(ICCD) camera (PI-MAX, Princeton instruments) were used to take discharge spectra. The camera is specified to have at most $5 \%$ non-linear intensity response which is critical for optical emission diagnostics such as excitation temperature of the plasma. The spectral intensity response of the spectrometer over 300-850 $\mathrm{nm}$ was corrected using a calibration lamp (LS-1-CAL, Ocean Optics). Three different crosssections of the plasma filament (denoted as A, B and C in Fig. 2) were imaged on the entrance slit of the spectrometer through a fused-silica lens with $2 \times$ magnification (Fig. 4). A variable aperture (nominally $2 \mathrm{~mm}$ ) was placed adjacent to the lens to minimize spherical and chromatic aberration. This improved the spectral resolution and the image focus over all of the experimentally-measured optical emission wavelengths.

For $\operatorname{Ar}\left(1 s_{5}\right)$ density and gas temperature, spatially resolved diode laser absorption spectrometry was used. A focused single-mode diode laser was passed through the cut-out shown in Figure 1 and scanned across the plasma in the T-line mode as seen in Figures $2 \mathrm{c}-2 \mathrm{e}$. Due to length limitations, the details of the absorption setup are described elsewhere [30].

\subsection{Microwave field simulation}

In this work, we wish to distinguish the differences between the SRR- and T-line modes. In particular, one must determine the (1) plasma impedance, (2) discharge current, (3) discharge voltage, and (4) actual plasma power for the two modes of operation. Therefore, the electromagnetic (EM) behavior of the plasma source is modeled using a 3-D simulator, HFSS ${ }^{\mathrm{TM}}$ (Ansoft Corp.) which uses the 


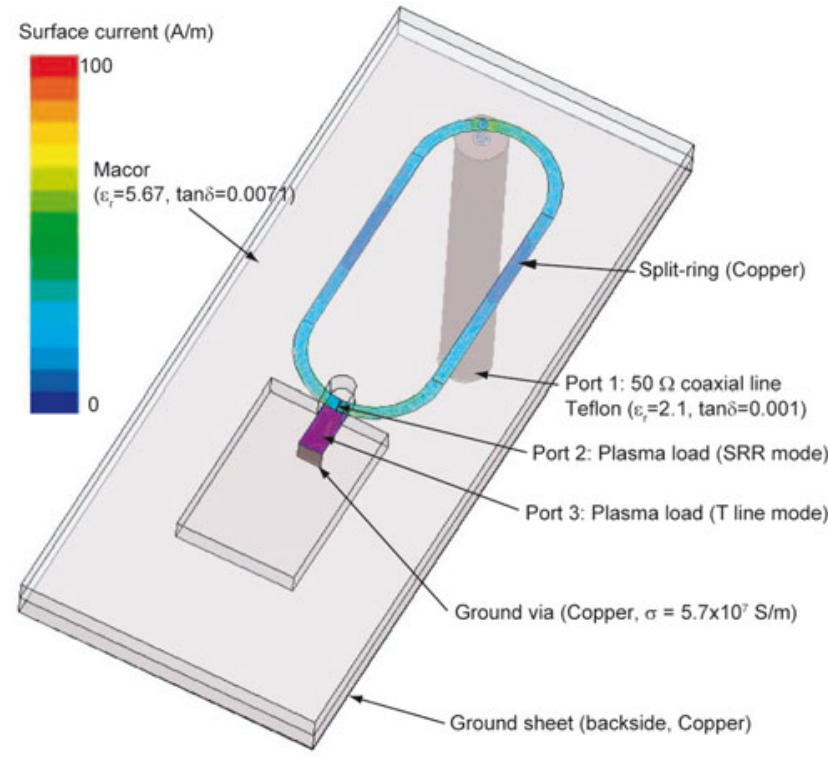

Fig. 5. (Color online) HFSS simulation model.

finite element method. Figure 5 shows the modeled geometry of the plasma source. HFSS computes the fields at specified driving frequencies, once the volume and boundary conditions are given. The EM properties such as permittivity, permeability, loss tangent and conductivity for each material were input as volume conditions. For the boundary condition, the outer boundaries of the box surrounding the device (not shown in the figure) were set as radiative, i.e. the waves were not reflected back to the device. Three electrical ports were defined in the model. Port 1 is the $50 \Omega$ co-axial line used as a power inlet. Port 2 is a rectangle region spanning the gap in the ring that simulates a plasma load in SRR mode. Port 3 is a rectangle from the split in the ring to the ground pin that simulates a plasma load in T-line mode. The impedances of port 2 and 3 were changed within HFSS to simulate various plasma conditions. For example, if both port 2 and port 3 are infinite impedances, the simulation solves the electromagnetic fields present prior to plasma ignition. The colormap on the split-ring in Figure 5 shows the magnitude of the surface current density in the T-line mode. In this particular case port 2 is set to model the minimum impedance of the SRR plasma $(100 \Omega)$ and port 3 represents the T-line discharge (typically $35 \Omega$ ).

\section{Microwave circuit analysis}

To investigate the transitions between the SRR and T-line modes, we begin by accurately measuring the forward and reflected power from the microplasma source. Figure 6 a shows the experimental power reflection coefficient $\left(P_{r e f} / P_{f w d}\right)$ as a function of forward power. Starting with only $15 \mathrm{~mW}$ of power, we observe that most of the forward power is absorbed by the device. This is expected because the SRR is designed to have an input impedance that matches the power source $(50 \Omega)$. As the forward power is increased, the discharge gap voltage increases to


Fig. 6. (Color online) Electrical characteristics of the SRR and T-line microdischarges: (a) measured microwave power reflection coefficient, (b) power dissipated in plasma, (c) plasma resistance $\left(R_{p}\right)$, (d) discharge voltage (zero-to-peak) and (e) discharge current (zero-to-peak). Quantities (b)-(e) are derived by matching the HFSS simulation to the reflection coefficient data of (a). The two hysteresis loops are defined by the arrows: (1) plasma ignition to SRR-mode, (2) transition from SRR- to T-line mode, and (3) transition from T-line mode to SRR-mode.

$150 \mathrm{~V}(0-\mathrm{pk})$ which subsequently ignites an argon plasma at $1 \mathrm{~atm}$. Ignition occurs at $0.7 \mathrm{~W}$ of forward power. The plasma is sustained in SRR mode while the forward power remains under $13 \mathrm{~W}$. Note that once the SRR-mode discharge is established, however, the power reflection coefficient instantly increases to well above 0.5. Any attempt to force the SRR plasma to absorb addition power results in a higher reflection coefficient. This is due to an impedance mismatch with the power supply that is induced by the low plasma resistance within the discharge gap. Decreasing the forward power to less than $0.7 \mathrm{~W}$, however, will 
decrease the reflection coefficient and allow the plasma to operate more efficiently, even down to 10's of $\mathrm{mW}$. In this manner, the resonator's impedance acts similar to a ballast. Any attempt to overheat the plasma with additional power results in more reflected power which then provides the critical reduction in the reduced electric field needed to prevent the ionization overheating instability. In fact, the discharge voltage remains nearly constant over a wide range of power (see Fig. 6d).

So far, the description above represents SRR behavior in the absence of the ground pin. With a current path to ground provided by this pin, a plasma can extend to the ground pin near $13 \mathrm{~W}$, creating the T-line mode. The plasma remains in this mode while the forward power is above $4 \mathrm{~W}$. We will show in the following sections that the T-line mode is a much more intense discharge. The high electron density of the T-line mode creates a low impedance discharge which optimally matches the device when $Z_{p}=35 \Omega$. Hence, the power reflection coefficient is observed to fall to $<0.1$ once the T-line discharge is established. In addition, the plasma power increases from an SRR-mode maximum of $1 \mathrm{~W}$ to approximately $10 \mathrm{~W}$ (Fig. 6b). As with much plasma behavior, the mode transition is hysteretic so arrows in Figure 6 show the direction of the mode transitions.

The measurements of the power reflection coefficient were next correlated to the plasma load impedance using the HFSS EM model. By systematically computing the reflection coefficients for all reasonable plasma load impedances, the plasma resistance was determined and reported in Figure 6c. In this method, the plasma impedance was assumed to be a real value, i.e. plasma resistance, $R_{p}$, although the actual impedance is generally a complex value. In order to find the imaginary part of $Z_{p}$, another experimental value is required such as the phase of the reflection coefficient. This measurement was not available, but the impedance of an atmospheric Ar plasma is dominated by resistance [10], and so the model's assumption is a good approximation to the actual discharge.

To simulate SRR mode operation, the port 2 resistance was modeled from $10^{2} \Omega$ to $10^{5} \Omega$ while keeping port 3 open. To simulate the T-line mode, the port 2 resistance was fixed at $10^{2} \Omega$ and port 3 resistance was changed from $1 \Omega$ to $10^{4} \Omega$.

Accurately correlating the measured powers with the model requires precise accounting of power loss due to finite conductivity, dielectric loss tangent, and radiation. These three terms allow one to find the efficiency with which power is coupled to the discharge from external measurements. HFSS computes power coupling between the three ports in our model in terms of $s$-parameters, such that

$$
\begin{aligned}
\left|s_{11}\right|^{2} & =\frac{P_{\text {ref }}}{P_{f w d}} \\
\left|s_{12}\right|^{2} & =\frac{P_{\text {plasma }}(\text { SRR mode })}{P_{f w d}} \\
\left|s_{13}\right|^{2} & =\frac{P_{\text {plasma }}(\text { T-line mode })}{P_{f w d}}
\end{aligned}
$$

口 Reflected power $\bigcirc$ Power into plasma $\triangle$ Loss
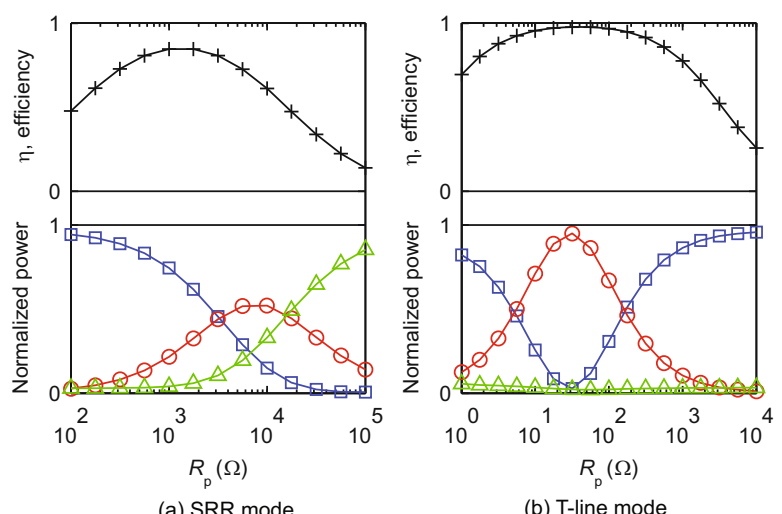

(b) T-line mode

Fig. 7. (Color online) Efficiency of power coupling to the discharge (top) and partitioning of forward power between the plasma, reflection, and loss (bottom) as a function of plasma resistance as simulated using HFSS: (a) SRR mode and (b) T-line mode. All data are normalized to $P_{f w d}=1 \mathrm{~W}$.

where $P_{f w d}, P_{\text {ref }}$ and $P_{\text {plasma }}$ are the forward power, reflected power, and power dissipated in plasma, respectively. The total power loss including the dielectric, conductor and radiation losses is determined from power conservation, such that

$$
\left|s_{11}\right|^{2}+\left|s_{12}\right|^{2}+\left|s_{13}\right|^{2}+\text { Loss }=1 .
$$

Due to the power loss, the power dissipated in the plasma is not exactly the forward minus reflected powers as is often assumed. The actual power delivered to the plasma can be expressed as,

$$
P_{\text {plasma }}=\left(P_{f w d}-P_{r e f}\right) \times \eta
$$

where $\eta$ is the power efficiency (which is load dependent) and can be estimated by equations (1)-(3), using

$$
\eta\left(R_{p}\right)=\frac{P_{\text {plasma }}}{P_{f w d}-P_{r e f}}=\left\{\begin{array}{l}
\frac{\left|s_{12}\right|^{2}}{1-\left|s_{11}\right|^{2}} \text { (SRR mode) } \\
\frac{\left|s_{13}\right|^{2}}{1-\left|s_{11}\right|^{2}}(\text { T-line mode })
\end{array}\right.
$$

Figure 7 (bottom) shows how the microwave power is partitioned using plasma resistance as a parameter. The input power is either reflected, dissipated in the plasma or lost as heat and radiation. Figure 7 (top) shows the corresponding efficiency of coupling power to the discharge, $\eta$. The loss in the SRR mode is found to monotonically increase as the plasma resistance increases. This is understood by noting that with large plasma resistance, the EM wave is highly reflected by the discharge in the gap. This means the average traveling distance of the wave - as it experiences multiple reflections from end-to-end along the SRR is quite long at resonance, and therefore the energy loss is large within the device. For low plasma resistances, the reflected power is high due to the impedance mismatch. The maximum power absorption occurs near $10 \mathrm{k} \Omega$, which is between these two extreme cases of the plasma resistance. 
Once the T-line mode is established, the loss is small (Fig. 7b) since the wave is either absorbed by the lowimpedance plasma or reflected back to the power supply without multiple internal reflections along the SRR. Therefore the wave's traveling distance is small, reducing loss. The maximum power is absorbed when the plasma resistance is equal to $35 \Omega$ which is the characteristic impedance of the nearly-shorted transmission line formed by the SRR. This result is as expected from the maximum power transfer theorem of transmission line theory.

Using the simulation results for efficiency from Figure 7 , the actual experimental power dissipated within the plasma and the plasma resistance were deduced as shown in Figures $6 \mathrm{~b}-6 \mathrm{c}$. The discharge voltage and current, $V_{0-p k}$ and $I_{0-p k}$, in Figures $6 \mathrm{~d}-6$ e were then obtained by,

$$
P_{\text {plasma }}=\frac{V_{0-p k}^{2}}{2 R_{p}}=\frac{R_{p} I_{0-p k}^{2}}{2} .
$$

The zero-to-peak discharge voltage was near $15 \mathrm{~V}$ and $35 \mathrm{~V}$ in SRR and T-line mode, respectively. The voltage of each discharge mode was nearly independent of the input power. The difference in discharge voltage between the two modes is likely dependent on the two different discharge gap lengths, 0.1 and $5 \mathrm{~mm}$, in the SRR and T-line modes. With increasing power, the plasma resistance decreases as expected, making a discontinuous transition to 35-ohms when the T-line mode ignited. Consequently, the discharge current increased with absorbed power. The current also exhibits a jump as the T-line mode begins. While the voltage is expected to depend on the particular gas, it is important to note that these microwave discharge voltages are an order of magnitude lower those found in DC microplasmas [1-6].

Finally, with the aid of the model we investigate our hypothesis that the SRR-mode discharge acts to shortcircuit the resonator. Figure 8 shows the instantaneous simulated voltage along the circumference of the splitring for three different load resistances $(100 \mathrm{k} \Omega, 1 \mathrm{k} \Omega$ and $100 \Omega$ ). With decreasing plasma resistance models, the magnitude of the voltage decreases as expected, provided that the power is held constant $\left(P_{f w d}=1 \mathrm{~W}\right)$. This prevents the reduced electric field from precipitating the IOI. As the voltage decreases, however, the phase of the discharge gap potential also transitions from nearly 180 degrees out of phase to in-phase. With low load resistance, the voltage across the SRR discharge gap becomes smaller than the voltage from the resonator to the ground pin. Using electrons or photoionization from the SRR discharge, the SRR mode triggers the T-line mode. It is necessary to note that the T-line mode is not able to ignite independently of the resonator-driven discharge due to the low electrode voltages associated with non-resonant wave transmission. Once established, however, the T-line mode improves the impedance mismatch inherent in the SRR mode, increases the absorbed power and creates a discharge that is an order of magnitude more dense as described next.

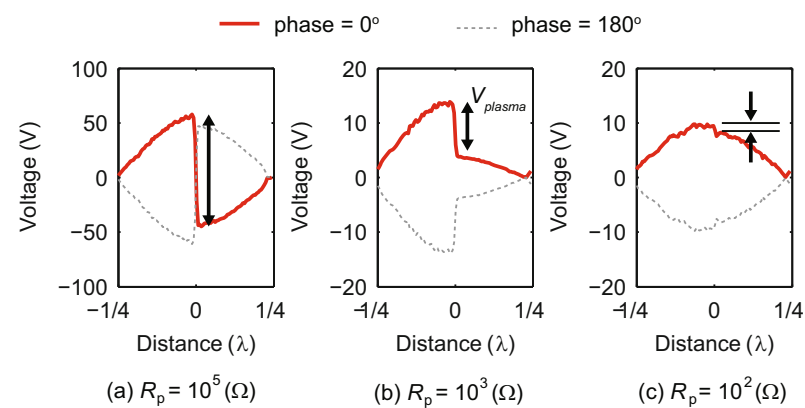

Fig. 8. (Color online) Simulated instantaneous voltage along the split-rings circumference in the SRR mode $\left(P_{f w d}=1 \mathrm{~W}\right)$. The discharge gap is located at $\lambda=0$. Small plasma resistance short-circuits the resonator, distorts the standing wave, and reduces the electrode potential (denoted by the arrows).

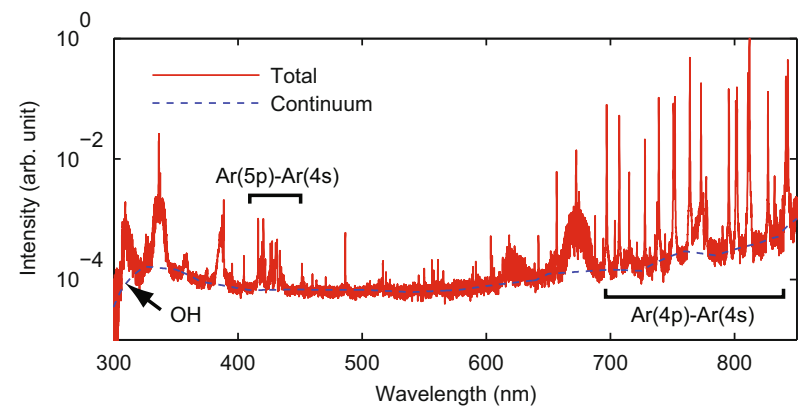

Fig. 9. (Color online) Emission spectrum of Ar plasma at atmospheric pressure.

\section{Optical plasma diagnostics}

\subsection{Emission spectrum}

Figure 9 shows an example of the experimental emission spectrum in the T-line mode. Continuum emission was observed in the entire experimental range of 300 to $850 \mathrm{~nm}$ as shown by the dashed line in Figure 9. The continuum is assumed to originate from e- $\mathrm{Ar}_{2}^{+}$recombination and the emission is proportional to electron density squared [31,33]. In experiments measuring the spectral line intensity, the continuum signal was first determined and subtracted. This subtraction is especially critical when estimating the excitation temperature which is sensitive to weak emission line intensities. Although the spectral response of the spectrometer has been calibrated, the calibration is rather uncertain under $350 \mathrm{~nm}$ and above $750 \mathrm{~nm}$ where the calibration lamp has a weak response.

\subsection{Rotational temperature}

The rotational temperature of $\mathrm{OH}$ molecules is estimated by fitting the experimental rotational emission spectrum in the 306 to $313 \mathrm{~nm}$ band with a synthetic spectrum as shown in Figure 10a. The synthetic spectrum was computed by LIFBASE [34] and it was convolved with the instrumental lineshape (see below). Rotational temperatures are often used as estimates of the gas temperature 
since the closely spaced rotational energy states are likely to be thermalized with the neutral gas at atmospheric pressure.

\subsection{Electron density}

Electron densities are estimated from the Stark broadening of $\mathrm{H}_{\beta}$ line emission at $486.1 \mathrm{~nm}$. Figure 10b shows the experimental spectrum. This emission spectrum is a convolution of instrument, Doppler, Van der Waals (collisional) and Stark broadening. The electron density is deduced once the instrumental broadening, the gas pressure and temperature are obtained.

The instrument broadening was determined by recording argon discharge emission lines at 0.5 torr. Under this condition, the electron density (Stark broadening) and pressure (collisional broadening) are low and Doppler broadening is dominant. Doppler is much smaller than the instrument broadening, however. The measured instrumental line shape was next fit with a Voigt profile to de-convolve into a Gaussian and a Lorentzian profile. The full-width at half maximum (FWHM) of the Gaussian and Lorentzian broadening of the instrument, $\Delta \lambda_{G i}$ and $\Delta \lambda_{L i}$ are,

$$
\begin{aligned}
\Delta \lambda_{G i} & =0.061 \mathrm{~nm} \\
\Delta \lambda_{L i} & =0.063 \mathrm{~nm} .
\end{aligned}
$$

Doppler broadening due to the motion of the emitter is Gaussian and the FWHM, $\Delta \lambda_{G d}$ is given by

$$
\Delta \lambda_{G d}=7.16 \times 10^{-7} \lambda \sqrt{\frac{T}{M}} \mathrm{~m}
$$

where $\lambda$ is the wavelength in meters, $T$ is the gas temperature in Kelvin and $M$ is the atomic mass of hydrogen atom in a.m.u. Here, the gas temperature was assumed to be equal to the measured rotational temperature of $\mathrm{OH}$ molecules.

Collisional broadening due to collisions between emitter $\left(\mathrm{H}^{*}\right)$ and neutral gas $(\mathrm{Ar})$ is Lorentzian and the FWHM, $\Delta \lambda_{L c}$ in $\mathrm{nm}$ is given by $[2]$

$$
\Delta \lambda_{L c}=6.8 \times 10^{-3} \frac{P}{T^{0.3}}
$$

where $P$ is the gas pressure in torr.

Stark broadening due to collisions between emitter $\left(\mathrm{H}^{*}\right)$ and charged species is Lorentzian and the FWHM, $\Delta \lambda_{L s}$ in $\mathrm{nm}$ is given by $[2,35]$

$$
\Delta_{L s}=1.92 \times 10^{-11} n_{e}^{2 / 3}
$$

where $n_{e}$ is the electron density in $\mathrm{cm}^{-3}$.

The total FWHM of the Gaussian component, $\Delta \lambda_{G}$ is given by

$$
\Delta \lambda_{G}=\sqrt{\Delta \lambda_{G i}^{2}+\Delta \lambda_{G d}^{2}} .
$$

The total FWHM of the Lorentzian component, $\Delta \lambda_{L}$ is given by

$$
\Delta \lambda_{L}=\Delta \lambda_{L i}+\Delta \lambda_{L c}+\Delta \lambda_{L s} .
$$

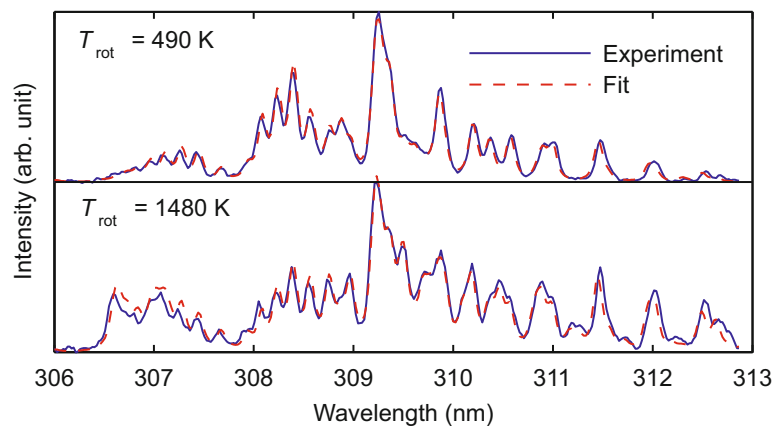

(a) $\mathrm{OH}$ rotational band spectrum

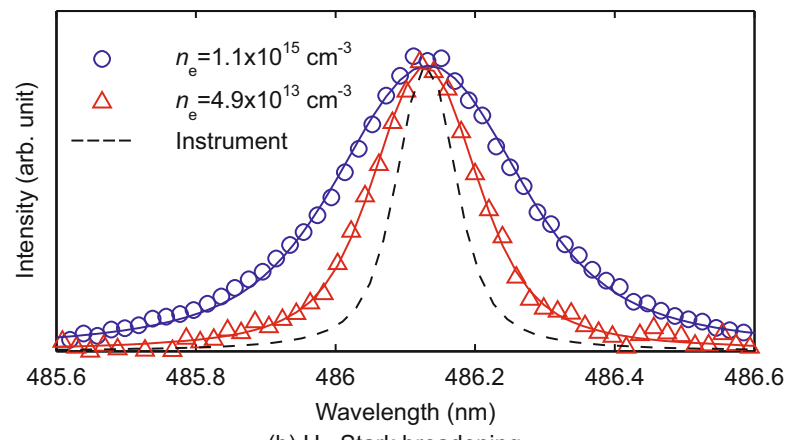

(b) $\mathrm{H}_{\beta}$ Stark broadening

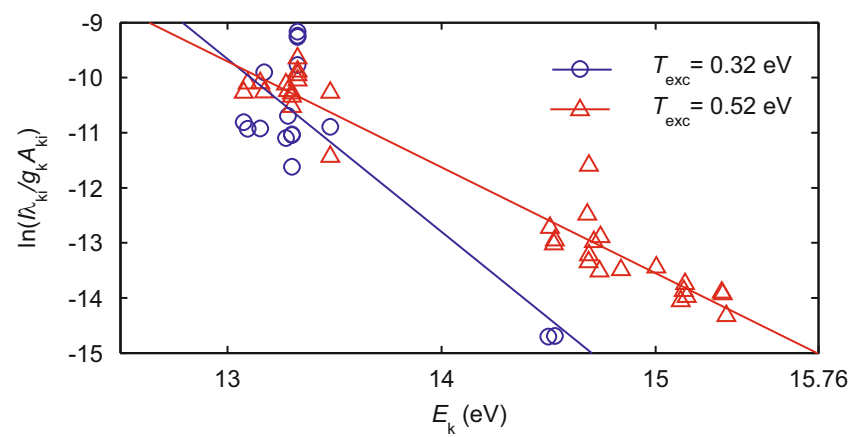

(c) Boltzmann plot

Fig. 10. (Color online) Typical argon microplasma characteristics at $1 \mathrm{~atm}$. of the SRR mode $\left(P_{\text {plasma }}=0.15 \mathrm{~W}\right)$ and Tline mode $\left(P_{\text {plasma }}=15 \mathrm{~W}\right)$. In each case, the lowest temperature or density in the figures corresponds to the SRR mode. (a) $\mathrm{OH}$ band spectra used for rotational temperature, (b) $\mathrm{H}_{\beta}$ line emission showing Stark broadening used for electron density and (c) Boltzmann plots of the Ar excited state populations used for excitation temperature.

The FWHM of the Voigt profile, $\Delta \lambda_{V}$ is approximated by $[36]$,

$$
\Delta \lambda_{V} \cong 0.5346 \Delta \lambda_{L}+\sqrt{0.2166 \Delta \lambda_{L}^{2}+\Delta \lambda_{G}^{2}} .
$$

Here $\Delta \lambda_{V}$ is the experimentally obtained FWHM estimated by fitting a Voigt profile to the emission spectrum, and $\Delta \lambda_{L s}$ is found by solving equations (8)-(15). Finally, the electron density is estimated from equation (12). 


\subsection{Excitation temperature}

Excitation temperature is a measure of the population distribution of Ar excited species ( $\left.\mathrm{Ar}^{*}\right)$. The expression $\frac{I_{k i} \lambda_{k i}}{g_{k} A_{k i}}$ is proportional to the upper state density of the transition, $N_{k}$, corrected for the degeneracy, $g_{k}$. The density distribution can often be approximated in Arrhenius form, such that [37]

$$
\ln \left(\frac{I_{k i} \lambda_{k i}}{g_{k} A_{k i}}\right)=-\frac{E_{k}}{k_{B} T_{e x c}}+C
$$

where the subscripts $k$ and $i$ denote the upper and lower states of the transition, $I_{k i}, \lambda_{k i}$ and $A_{k i}$ are the emission intensity, the wavelength and the Einstein coefficient of the transition, $k_{B}$ is the Boltzmann constant, $E_{k}$ is the upper state energy level, $T_{\text {exc }}$ is the excitation temperature and $C$ is a constant. The atomic emission constants are obtained from the NIST database ${ }^{1}$. The Boltzmann plot consists of $\frac{I_{k i} \lambda_{k i}}{g_{k} A_{k i}}$ plotted against $E_{k}$ and the excitation temperature is obtained from the slope of the linear fit as shown in Figure 10c.

\section{5 $\operatorname{Ar}\left(1 \mathrm{~s}_{5}\right)$ absorption}

The density and temperature of the Ar metastable state $\left(1 s_{5}\right)$ was found by optical absorption of using the $801.4 \mathrm{~nm}\left(1 s_{5}-2 p_{8}\right)$ transition. By using a highly focused laser, the spatially resolved line-integrated density and gas temperature were estimated following a previously published method $[30,32]$. The line-integrated density of $\operatorname{Ar}\left(1 s_{5}\right)$ was found from the total integrated absorption of laser photons as the wavelength of the laser was scanned through the transition wavelength. The gas temperature was estimated from the line broadening. At atmospheric pressure, collisional broadening is the dominant mechanism observed and the Doppler broadening in equation (10) has a neglible effect. The FWHM of the collisional broadening, $\Delta \lambda_{c}$ is given by,

$$
\Delta \lambda_{c}=C_{c} \frac{P}{k_{B} T^{0.7}}
$$

where $C_{c}$ is the collisional broadening parameter. Although both Doppler and collisional broadening are functions of the gas temperature, the gas temperature is the only unknown and so the gas temperature may be estimated from the absorption line broadening. The method is somewhat uncertain, however, because the reported collisional broadening parameters, $C_{c}$ have rather large discrepancies. Vallee et al. reported $C_{c}=1.51 \times$ $10^{-37} \mathrm{~m}^{4} \mathrm{~K}^{-0.3}$ at $T=3900 \mathrm{~K}[38]$. In our previous work [32], the measured value was $C_{c}=2.54 \times$ $10^{-37} \mathrm{~m}^{4} \mathrm{~K}^{-0.3}$ at $T=340 \mathrm{~K}$. In this work, the reported gas temperature by laser diode absorption was comparable to the $\mathrm{OH}$ rotational temperature if the value of Vallee et al. was used.

\footnotetext{
1 NIST Atomic Spectra Database, http://physics.nist. gov/PhysRefData/ASD/index.html
}
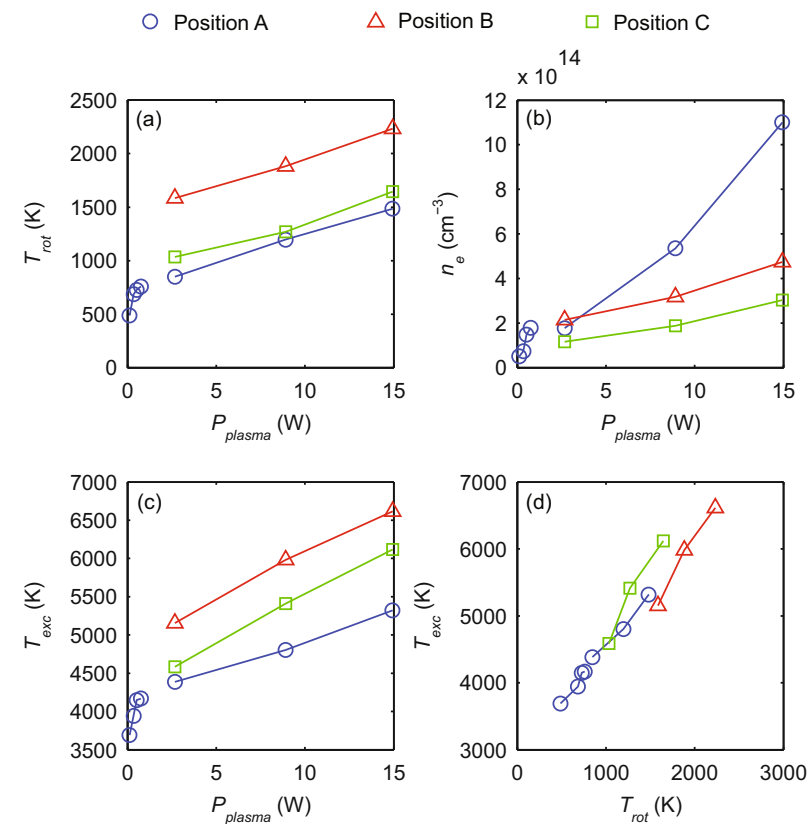

Fig. 11. (Color online) Plasma parameters measured in the central core using spatially-resolved optical diagnostics: (a) $\mathrm{OH}$ rotational temperature, (b) electron density, (c) excitation temperature and $(\mathrm{d})$ correlation between rotational and excitation temperatures.

\subsection{Results}

Imaging spectra were taken across the $x$-direction of the microplasma following lines A, B and C as denoted in Figures $1-2$. In the SRR mode, please note that the plasma was sustained only at position A. The line-integrated plasma parameters, described in the previous section, were calculated along the $x$-direction. Often the maximum values are found in the central core of the microplasma and these are plotted in Figures 11a-11c. The axes of the subfigures are the actual power dissipated in the plasma, including efficiency and loss, as estimated by the method described in Section 3.

The $\mathrm{OH}$ rotational temperature in the SRR mode changed from $490 \mathrm{~K}$ to $760 \mathrm{~K}$ with increasing power. In the T-line mode, the temperature is much higher $(850 \mathrm{~K}$ to $1480 \mathrm{~K}$ ) at position A. Notably, the rotational temperature is even hotter still ( $1580 \mathrm{~K}$ to $2230 \mathrm{~K})$ at position $\mathrm{B}$. The temperature in the middle of the microdischarge (position B) is higher than at either position $\mathrm{A}$ or $\mathrm{C}$ where the plasma is closer to a solid surface. This indicates that heat conduction due to the high internal temperature gradient is an important heat loss mechanism. In the T-line mode, the electron density increased from $1.8 \times 10^{14} \mathrm{~cm}^{-3}$ to $1.1 \times 10^{15} \mathrm{~cm}^{-3}$ with increasing power. The electron density was the highest at position A where the plasma is most confined and the lowest at position $\mathrm{C}$ where the plasma is more diffuse as shown in Figure 2. Hrycak et al. reported a comparable electron density of $1.4 \times 10^{15} \mathrm{~cm}^{-3}$ at $15 \mathrm{~W}$ in argon, using a $2.45 \mathrm{GHz}$ co-axial waveguide needle type source [17]. The excitation temperature shown in Figure 11c has a similar 
trend to the rotational temperature. Both plasma properties show a significant increase between the SRR and Tline modes. Figure 11d shows the correlation between the rotational and excitation temperatures, and this demonstrates a more or less linear dependence. The SRR mode maintains a stable, relatively cold microplasma for absorbed powers less than a few watts. The transition to the T-line mode, however, exhibits a much more dense plasma with higher gas temperatures. Although approaching the characteristics of an arc, the T-line mode discharge does not melt the copper electrodes. We believe that the Tline mode, while no longer ballasted by the resonator, is controlled against IOI by limiting the power from the microwave amplifier. Given sufficient input power, however, trends indicate that the T-line microplasma would eventually create a thermal arc.

The previous section discusses the plasma parameters that were measured in the central core of both SRR and T-line mode microplasmas. The intensity of the T-line mode, however, warrants further investigation of the spatially-resolved profiles. Figure $12 \mathrm{a}$ shows the line-integrated emission and absorption of various excited species across the width of the plasma filament in T-line mode at position B. All data are for a high power input of $P_{\text {plasma }}=15 \mathrm{~W}$. Assuming the plasma is cylindrically symmetric at this position, the line-integrated profiles were also Abel inverted to reveal the local emission and absorption. The inverted profiles are plotted with dashed lines in Figure 12a and show that some species are heavily depleted from the core region. The cylindrical approximation has not been rigorously validated, so the inverted profiles give a coarse estimate of the interior species behavior. The details of Abel inversion and the internal structure of the low-power SRR mode are described elsewhere [30]. As seen in the figure, the various species have clearly different spatial distributions. Electrons and argon ions have center-peaked profiles. On the other hand, $\mathrm{OH}$ and $\operatorname{Ar}\left(1 s_{5}\right)$ excited states have centerdepleted profiles similar to previous observations of the SRR microplasma. Due to this center-depletion, $\mathrm{OH}$ emission and $\operatorname{Ar}\left(1 s_{5}\right)$ absorption spectra are not good indicators of the central gas temperature. This phenomenon results in an overly flat temperature profile measurement near the center as plotted in Figure 12b. The actual temperature profile is expected to be center-peaked, since the gas heating originates from the centrally-peaked charged species and diffuses outward. The true center temperature is estimated to be above $3000 \mathrm{~K}$ for this particular case. The core temperature was estimated from the wings of the temperature profile, assuming the radial temperature profile was similar to the one previously simulated with a simple heat transfer model for the SRR-mode [30]. Therefore, the line-integrated measurement of the $\mathrm{OH}$ rotational temperature underestimates the center temperature. The excitation temperature is primarily deduced from short-lived excited states $(\operatorname{Ar}(4 p)$ and $\operatorname{Ar}(5 p))$ that are centrally-peaked. Therefore the spatial distribution of the excitation temperature (Fig. 12c) is believed to be more representative of the actual plasma core.

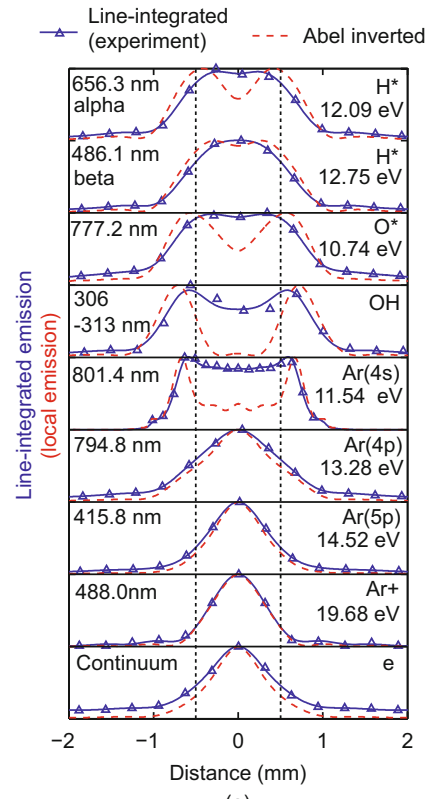

(a)

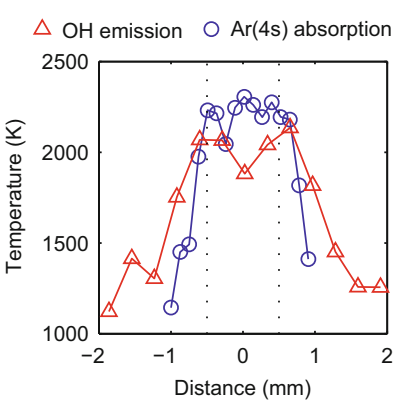

(b)

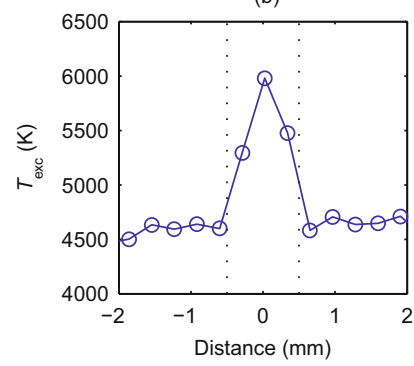

(c)
Fig. 12. (Color online) (a) The spatially-resolved emission intensities of various species measured across the discharge at position B (Abel inverted profiles are shown as dashed lines). The $\operatorname{Ar}\left(1 s_{5}\right)$ metastable density profile is obtained by laser diode absorption. (b) A comparison of spatially-resolved gas temperature estimated by the $\mathrm{OH}$ rotational temperature at position $\mathrm{B}\left(P_{\text {plasma }}=9 \mathrm{~W}\right)$ and by $\operatorname{Ar}\left(1 s_{5}\right)$ absorption linewidths measured through the cut-out near position B $\left(P_{\text {plasma }}=6.5 \mathrm{~W}\right)$. (c) Spatial variation of excitation temperature at position $\mathrm{B}$ $\left(P_{\text {plasma }}=9 \mathrm{~W}\right)$.

The maximum gas temperature in T-line mode was estimated to exceed $3000 \mathrm{~K}$ at the core. Ion Joule heating near the sheath should be minimal due to the low applied voltage. The high temperature of the $5 \mathrm{~mm}$-long T-line mode discharge is partially due to slow heat conduction through the relatively long distance from the plasma's core to the ground electrode. There is also believed to be considerable heat generation from sources other than ion Joule heating such as e-Ar momentum transfer or e- $\mathrm{Ar}_{2}^{+}$ dissociative recombination which couple kinetic energy to Ar atoms in atmospheric discharges [39].

Finally, the $\mathrm{H}_{\beta}$ line profile is also found to be centrallypeaked and is slightly broader than the continuum (electron) distribution. It is therefore believed that the $\mathrm{H}_{\beta}$ Stark broadening measurement is a reasonable determination of core electron density. Note, however, that the $\mathrm{H}_{\alpha}$ emission is slightly center-depleted and therefore may provide less accurate peak electron density data.

\section{Conclusion}

In this work the inherent stability of resonator-driven microplasmas is investigated. Even if microwave power exceeding $10 \mathrm{~W}$ is applied to a split-ring resonator (SRR) with a $0.1 \mathrm{~mm}$ discharge gap, the severe plasma loading of the resonator causes large reflected power. In this manner, 
the power absorbed by the discharge is limited and the sudden drop in plasma resistance due to the ionization overheating instability is avoided as the resonator circuit rejects excess power.

A modified SRR plasma source is shown to operate in two modes: a low density SRR mode and a high density T-line mode. The addition of a ground electrode in the vicinity of the SRR discharge gap allows the sudden transition to the high density state. Using microwave circuit analysis, we report the plasma resistance, discharge voltage and discharge current in both modes. As expected, the high density T-line mode exhibits a low plasma resistance. Unlike the SRR mode which couples power to the plasma most efficiently for $R_{p}$ on the order of $10 \mathrm{k} \Omega$, the T-line mode optimally couples power to the plasma when the plasma impedance matches that of the transmission line (typically $35 \Omega$ ). In addition, plasma parameters such as the gas temperature, electron density and excitation temperature were measured by optical emission. These diagnostics reveal a substantial increase in plasma density and temperature when the plasma transitions from SRR mode to T-line mode. The sudden change in plasma parameters, however, is clearly related to the jump in discharge power made possible by the ground path in the T-line mode.

The estimated discharge voltages were low: $15 \mathrm{~V}$ in SRR mode and $35 \mathrm{~V}$ in the T-line mode. The low electrode voltages limit the ionization energy available from secondary electron emission at the electrodes surface. In contrast, one observes that $13.56 \mathrm{MHz}$ rf capacitive microdischarges have much larger electrode potentials. Those high voltage microdischarges may have stability issues due to the transition from the $E$-field driven $\alpha$ mode to the secondary electron driven $\gamma$ mode [40]. In this work, only the $\alpha$ discharge mode was observed and there is never an intense plasma layer near the electrodes due to secondary electrons. Therefore both the SRR and T-line modes were free of stability issues due to energetic secondary electrons.

This work was supported in part by the U.S. Department of Energy under award No. DE-SC0001923 and by the National Science Foundation under Grant CBET-0755761.

\section{References}

1. V. Arkhipenko, A. Kirillov, Y. Safronau, L. Simonchik, Eur. Phys. J. D 60, 455 (2010)

2. S. Belostotskiy, T. Ouk, V. Donnelly, D. Economou, N. Sadeghi, J. Appl. Phys. 107, 053305 (2010)

3. Q. Wang, I. Koleva, V. Donnelly, D. Economou, J. Phys. D 38, 1690 (2005)

4. D. Staack, B. Farouk, A. Gutsol, A. Fridman, Plasma Sources Sci. Technol. 14, 700 (2005)

5. M. Kushner, J. Phys. D 38, 1633 (2005)

6. K. Schoenbach, M. Moselhy, W. Shi, Plasma Sources Sci. Technol. 13, 177 (2004)

7. K. Takaki, M. Hosokawa, T. Sasaki, S. Mukaigawa, T. Fujiwara, Appl. Phys. Lett. 86, 151501 (2005)

8. J. Walsh, F. Iza, M. Kong, Eur. Phys. J. D 60, 523 (2010)
9. A. Kono, J. Wang, M. Aramaki, Thin Solid Films 506, 444 (2006)

10. F. Iza, J. Hopwood, Plasma Sources Sci. Technol. 14, 397 (2005)

11. F. Iza, J. Hopwood, IEEE Trans. Plasma Sci. 33, 306 (2005)

12. F. Iza, J. Hopwood, IEEE Trans. Plasma Sci. 32, 498 (2004)

13. F. Iza, J. Hopwood, IEEE Trans. Plasma Sci. 31, 782 (2003)

14. J. Xue, J. Hopwood, IEEE Trans. Plasma Sci. 37, 816 (2009)

15. Z. Zhang, J. Hopwood, Appl. Phys. Lett. 95, 161502 (2009)

16. J. Gregório, L. Alves, O. Leroy, P. Leprince, C. BoisseLaporte, Eur. Phys. J. D 60, 627 (2010)

17. B. Hrycak, M. Jasiński, J. Mizeraczyk, Eur. Phys. J. D 60, $606(2010)$

18. L. Zhu, W. Chen, X. Zhu, Y. Pu, Z. Li, Rev. Sci. Instrum. 80, 023105 (2009)

19. X.M. Zhu, W.C. Chen, Y.K. Pu, J. Phys. D: Appl. Phys. 41, 105212 (2008)

20. J. Kim, K. Terashima, Appl. Phys. Lett. 86, 191504 (2005)

21. D. Staack, B. Farouk, A. Gutsol, A. Fridman, J. Appl. Phys. 106, 013303 (2009)

22. D. Hsu, D. Graves, J. Phys. D 36, 2898 (2003)

23. K. Schoenbach, A. El-Habachi, W. Shi, M. Ciocca, Plasma Sources Sci. Technol. 6, 468 (1997)

24. U. Kogelschatz, Plasma Chem. Plasma Proc. 23, 1 (2003)

25. J. Waskoenig, D. O'Connell, V. Schulz-Von Der Gathen, J. Winter, S. Park, J. Eden, Appl. Phys. Lett. 92, 101503 (2008)

26. S. Starostin, M. ElSabbagh, E. Aldea, H. de Vries, M. Creatore, M. van de Sanden, IEEE Trans. Plasma Sci. 36, 968 (2008)

27. S. Starostin, P. Premkumar, M. Creatore, E. Van Veldhuizen, H. De Vries, R. Paffen, M. Van de Sanden, Plasma Sources Sci. Technol. 18, 045021 (2009)

28. O. Sakai, T. Sakaguchi, Y. Ito, K. Tachibana, Plasma Phys. Control. Fusion 47, B617 (2005)

29. K. Urabe, O. Sakai, K. Tachibana, J. Phys. D 44, 115203 (2011)

30. N. Miura, J. Hopwood, J. Appl. Phys. 109, 013304 (2011)

31. N. Miura, J. Hopwood, J. Appl. Phys. 109, 113303 (2011)

32. N. Miura, J. Xue, J. Hopwood, IEEE Trans. Plasma Sci. 38, $2458(2010)$

33. A. Lissovski, A. Treshchalov, Phys. Plasmas 16, 123501 (2009)

34. J. Luque, D. Crosley, SRI International Report MP 99-009, 1999

35. H. Griem, Phys. Rev. 128, 515 (1962)

36. J. Olivero, R. Longbothum, J. Quant. Spectrosc. Radiat. Transfer 17, 233 (1977)

37. W. Wiese, Spectrochim. Acta B 46, 831 (1991)

38. O. Vallee, P. Ranson, J. Chapelle, J. Quant. Spectrosc. Radiat. Transfer 18, 327 (1977)

39. G. Ramos, M. Schlamkowitz, J. Sheldon, K. Hardy, J. Peterson, Phys. Rev. A 51, 2945 (1995)

40. J. Laimer, H. Störi, Plasma Process. Polym. 3, 573 (2006)

Open Access This is an open access article distributed under the terms of the Creative Commons Attribution License (http://creativecommons.org/licenses/by/3.0), which permits unrestricted use, distribution, and reproduction in any medium, provided the original work is properly cited. 